\title{
Mitral valve repair for endocarditis
}

\author{
Silvia Solari ${ }^{1}$, Emiliano Navarra ${ }^{2}$, Laurent de Kerchove ${ }^{2}$, and Gebrine Elkhoury ${ }^{2}$ \\ ${ }^{1}$ Cliniques de l'Europe - site Sainte Elisabeth \\ ${ }^{2}$ Cliniques universitaires Saint-Luc
}

August 12, 2020

\begin{abstract}
Many authors have reported their results of mitral valve (MV) repair1,2 in acute and healed endocarditis. However, the results published from different authors highlight the fact that the reparability rate for this indication remains low. Over the last 3 decades our group has adopted an early and repair-oriented approach to the infective endocarditis with the objective to improve the repair rate and the long-term results. In this paper we describe our institutional experience on mitral valve repair for infective endocarditis. Data for this paper were extracted from our institutional database on heart valve disease. From 1991 to 2015, 160 consecutive patients in our institution underwent MV surgery for active IE on native MV. The median follow-up was 122 months. This study was approved by the institutional ethics review board, and written informed consent was waived for this study given its retrospective design. Hospital mortality was $11.6 \%(\mathrm{n}=18)$. Early MV reoperation before hospital discharge was required in $5(3.1 \%)$ patients. At 5, 10 and 15 years, overall survival in the MVr for endocarditis in group was $79 \pm 4 \%, 65+-5 \%, 57+-6 \%$, respectively. Freedom from reoperation at 5, 10 and 15 years was $95+-2 \%, 88+-4 \%$ and 81 +- $6 \%$, respectively. Mitral infective endocarditis is an insidious pathology and his surgical approach can be challenging. An early and repair-oriented surgical approach can allow to improve reparability rates with good long-term durability and a low recurrence rate of endocarditis.
\end{abstract}

\section{Hosted file}

NOTABLESMitral valve repair for endocarditis-Journal of Cardiac Surgery.pdf available at https://authorea.com/users/350394/articles/475250-mitral-valve-repair-for-endocarditis 


\begin{tabular}{|l|l|l|}
\hline & \multicolumn{2}{|c|}{ MVRepair (n=155) } \\
\hline Echo findings & 87 & $55.4 \%$ \\
\hline Vegetation(s) $>1 \mathrm{~cm}$ & 44 & $28.0 \%$ \\
\hline Abscess & 87 & $55.4 \%$ \\
\hline MR 2-3/4 & 76 & $34.4 \%$ \\
\hline Embolic complications & 31 & $19.7 \%$ \\
\hline Antimicrobial drugs resistance & 35 & $22.3 \%$ \\
\hline Heart failure
\end{tabular}




\begin{tabular}{|l|c|c|}
\hline & \multicolumn{2}{|c|}{ MVRepair $\mathbf{n = 1 5 5}$} \\
\hline No patch techniques & $\mathrm{n}=65$ & $41.9 \%$ \\
\hline Triangular or quadrangular resection & 65 & $41.9 \%$ \\
\hline Quadrangular resection + sliding plasty & 18 & $11.6 \%$ \\
\hline Artificial neochordae (Goretex ${ }^{\circledR}$ CV5) & 23 & $14.8 \%$ \\
\hline Native chordae transfert & 13 & $8.4 \% \%$ \\
\hline Commisurotomy & 3 & $1.9 \% \%$ \\
\hline Papillary muscles shortening & 1 & $0.6 \%$ \\
\hline & & \\
\hline Patch techniques & $\mathrm{n}=90$ & $58.1 \%$ \\
\hline Pericardial patch (bovine et autologous) ${ }^{*}$ & 72 & $46.5 \%$ \\
\hline Tricuspid autograft patch & 9 & $5.8 \%$ \\
\hline Flip-over technique (transfert from the posterior to the anterior & 7 & $4.5 \%$ \\
\hline leaflet) & 11 & $7.1 \%$ \\
\hline Part of a mitral valve homograft & 79 & $51 \%$ \\
\hline Annuloplasty techniques & 63 & $40.6 \%$ \\
\hline Prosthetic ring & 16 & $10.3 \%$ \\
\hline Pericardial Band & & \\
\hline Prosthesis & - & - \\
\hline Biological prosthesis & - & - \\
\hline Mechanical prosthesis & & \\
\hline & \multicolumn{2}{|c|}{$128 \pm 51.2$} \\
\hline ECC mean time (min)** & \multicolumn{2}{|c|}{$90 \pm 31.3$} \\
\hline Aortic cross clamp mean time (min) & \multicolumn{2}{|c|}{-} \\
\hline
\end{tabular}

